\title{
Spectrum of histologic changes in colonic biopsies in patients treated with mycophenolate mofetil
}

\author{
Megan K Selbst ${ }^{1}$, William A Ahrens ${ }^{1}$, Marie E Robert ${ }^{1}$, Amy Friedman ${ }^{2}$, Deborah D Proctor ${ }^{3}$ \\ and Dhanpat Jain ${ }^{1}$ \\ ${ }^{1}$ Department of Pathology, Yale University School of Medicine, New Haven, CT, USA; ${ }^{2}$ Department of Surgery, \\ Yale University School of Medicine, New Haven, CT, USA and ${ }^{3}$ Section of Digestive Diseases, Department of \\ Internal Medicine, Yale University School of Medicine, New Haven, CT, USA
}

\begin{abstract}
Mycophenolate mofetil, an immunosuppressive agent, is frequently used following bone marrow and solid organ transplantation. Diarrhea is a commonly seen side effect of mycophenolate mofetil, which may necessitate colonic biopsy in some patients. The histologic changes found in this setting have been reported to mimic self-limited colitis, graft-vs-host disease or inflammatory bowel disease in isolated case reports, and could pose diagnostic and management difficulties. The goal of this study is to define the spectrum of histologic changes in colonic biopsies associated with mycophenolate mofetil usage. All solid organ transplant patients who received mycophenolate mofetil and underwent colonic biopsy for gastrointestinal symptoms from 1999 to 2007 were included in the study. Patients who did not receive mycophenolate mofetil were used as controls. Various histologic features including architectural distortion, apoptosis, inflammatory infiltrate, Paneth cell metaplasia and mucin depletion were subjectively evaluated and scored (scale: 0-3) by two independent reviewers in a blinded fashion. Forty solid organ transplant patients underwent colonic biopsy for gastrointestinal symptoms during the study period. Biopsies from $69 \%$ of patients on mycophenolate mofetil showed histologic changes. Apoptosis $(41 \%)$ and architectural distortion $(66 \%)$ were seen more frequently in patients receiving mycophenolate mofetil as compared to the control group (13\%). The histologic changes in patients receiving mycophenolate mofetil were categorized as normal/near normal (31\%), inflammatory bowel disease-like (28\%), graft-vs-host disease-like $(19 \%)$, ischemia-like $(3 \%)$ and self-limited colitis-like $(16 \%)$ changes. Of the controls, only one patient showed a graft-vs-host disease-like histologic pattern. In conclusion, histologic changes are frequently associated with mycophenolate mofetil use and can resemble self-limited colitis, graft-vs-host disease and inflammatory bowel disease leading to diagnostic difficulties. Increased awareness of the histologic spectrum of mycophenolate mofetil-induced changes is required by the pathologist to avoid diagnostic errors.
\end{abstract}

Modern Pathology (2009) 22, 737-743; doi:10.1038/modpathol.2009.44; published online 27 March 2009

Keywords: mycophenolate mofetil; colitis; diarrhea

Mycophenolate mofetil, an immunosuppressive agent, was FDA-approved in early 1995 for prophylaxis of organ rejection in patients undergoing allogeneic renal or cardiac transplantation, and in 2000 to prevent liver transplant rejection. ${ }^{1}$ The introduction of this drug has led to a significant decrease in the incidence of acute allograft rejection in solid organ transplant patients. ${ }^{2}$ Mycophenolate

Correspondence: Dr D Jain, MD, Department of Pathology, Yale University School of Medicine, 310 Cedar Street, PO Box 208023, New Haven, CT 06520-8023, USA.

E-mail: Dhanpat.jain@yale.edu

Received 7 October 2008; revised and accepted 22 December 2008; published online 27 March 2009 mofetil is a prodrug of mycophenolic acid (MPA), an antibiotic substance derived from Penicillium stoloniferum. By inhibiting inosine monophosphate dehydrogenase, an enzyme in the de novo pathway of purine synthesis, MPA prevents the proliferation of $\mathrm{T}$ and $\mathrm{B}$ lymphocytes and the formation of antibodies from B cells. It also may inhibit recruitment of leukocytes to inflammatory sites. ${ }^{3}$

Mycophenolate mofetil is currently used in treatment regimes as an immunosuppressive agent in both solid organ and bone marrow/peripheral blood stem cell transplantation, as well as in treatment of autoimmune disorders, such as lupus. Common gastrointestinal side effects of mycophenolate mofetil ( $45 \%$ of patients) include diarrhea, nausea, 
vomiting, abdominal pain and gastritis. ${ }^{2,4}$ These complications range from mild (intermittent diarrhea or nausea) to life threatening (colonic necrosis or perforation). ${ }^{4}$ Often the clinical differential diagnosis of gastrointestinal symptoms includes infections, graft-vs-host disease and other defined inflammatory disorders of the gastrointestinal tract, eg, chronic idiopathic inflammatory bowel disease. Differentiation among these conditions is difficult on clinical grounds and biopsies are often performed to clarify the diagnosis. An accurate diagnosis is essential in these patients due to the differences in treatment for each condition. Although dose reduction due to drug toxicity may relieve symptoms, it may also increase the risk of acute rejection and have an adverse impact on longterm graft survival. ${ }^{5}$

Histologic changes seen in patients with mycophenolate mofetil-associated drug toxicity have been reported to mimic graft-vs-host disease, ${ }^{6}$ inflammatory bowel disease, ${ }^{7}$ ischemia $^{8}$ or acute self-limited colitis in isolated case reports. The histologic similarities with other conditions can pose diagnostic and management difficulties, particularly in allogeneic bone marrow/peripheral blood stem cell transplantation when graft-vs-host disease is in the clinical differential diagnosis. Although graft-vs-host disease is a well-recognized and not infrequent complication of allogeneic bone marrow/ peripheral blood stem cell transplantation, it is much less common in solid organ transplantation (eg, liver, kidney and heart). Therefore, patients with solid organ transplantation represent a reasonable population to study the histologic changes of mycophenolate mofetil. The goal of this study was to define the spectrum of histologic changes in colonic biopsies associated with the use of mycophenolate mofetil. Bone marrow/peripheral blood stem cell transplant patients were excluded to avoid confusion between graft-vs-host disease and mycophenolate mofetil toxicity.

\section{Materials and methods}

All solid organ transplant patients who were receiving mycophenolate mofetil and had colonic biopsies performed for gastrointestinal symptoms between 1999 and 2007 were identified from a search of the pathology database and included in the study. Transplant patients who did not receive mycophenolate mofetil during the same period were used as controls. Bone marrow/peripheral blood stem cell transplant patients were excluded to avoid uncertainty of the final diagnosis with regards to graft-vs-host disease and mycophenolate mofetil toxicity. Patients with known active viral infections (CMV, HIV and HSV) or a preexisting diagnosis of inflammatory bowel disease were also excluded. The possibility of graft-vs-host disease was considered low in the clinical differential diagnosis and was further excluded by clinical follow-up in all patients. The following histologic features were evaluated and scored subjectively on a scale from 0 to 3: architectural distortion, crypt cell apoptosis ( $>5 / 100$ crypts), chronic or acute inflammatory infiltrate, mucin depletion, crypt loss, cryptitis and Paneth cell metaplasia. Apoptosis was graded as 0: $<5 / 100$ crypts, $1+: 5-10 / 100$ crypts, $2+: 11-20 /$ 100 crypts and $3+:>21 / 100$ crypts. On the basis of the overall histology, the cases were placed in one of the following categories: normal/near normal, ischemia-like, inflammatory bowel disease-like, graft-vshost disease-like and self-limited colitis-like. Changes were considered ischemia-like when the biopsy showed mucin-depleted crypts with preserved crypt architecture, lack of significant lamina propria inflammation ( 0 to $1+$ ) and crypt dropout with or without erosions. The pattern was considered inflammatory bowel disease-like when the biopsy showed moderate to marked crypt architectural distortion ( 2 to $3+$ ) with increased lamina propria lymphoplasmacytic infiltrate $(1$ to $3+$ ). The changes were considered graft-vs-host disease-like when there was increased enterocyte apoptosis in the absence of any lamina propria inflammation and lack of significant crypt architectural distortion. Minimal crypt distortion ( 0 to $1+$ ) or rare branching crypts were considered acceptable for the graft-vshost disease-like pattern. Presence of neutrophilpredominant lamina propria infiltrate with cryptitis/crypt abscesses in the absence of crypt architectural distortion was considered a self-limited colitislike pattern. Mild enterocyte apoptosis $(1+)$ was considered acceptable with other patterns (inflammatory bowel disease-like, self-limited colitis-like or ischemia-like). The clinical history, including the presenting symptoms, medications, including immunosuppressive drugs, colonoscopic findings, bowel preparation method and follow-up, was obtained.

\section{Results}

A total of 40 solid organ transplant patients underwent colonic biopsy for gastrointestinal symptoms during the study period, of which 32 patients were taking mycophenolate mofetil at the time of biopsy (dosages ranged from 500 to $1000 \mathrm{mg}$ twice daily). The transplants in the mycophenolate mofetil group consisted of kidney (17), kidney/pancreas (5), heart (6), heart/kidney (3) and liver (1). The transplants in patients not taking mycophenolate mofetil included kidney (6), kidney/pancreas (1) and liver (1). The bowel preparation methods before the colonoscopy included GoLytely 16 (50\%), phospho soda $(2 / 32,6 \%)$, tap water enema $(1 / 32,3 \%)$ and Fleets enema $4(12.5 \%)$. Two patients had no bowel preparation $(2 / 32,6 \%)$. The bowel preparation information in the remaining 7 cases was not available $(7 / 32,22 \%)$ (Table 1$)$. 
Table 1 Pattern of endoscopic changes and bowel preparation methods and their correlation with histologic patterns

\begin{tabular}{|c|c|c|c|c|c|c|}
\hline & $\begin{array}{c}\text { Normal/near } \\
\text { normal }(\mathrm{n}=9)\end{array}$ & $\begin{array}{c}I B D \\
(\mathrm{n}=9)\end{array}$ & $\begin{array}{l}G V H D \\
(\mathrm{n}=6)\end{array}$ & $\begin{array}{c}\text { Ischemia } \\
(\mathrm{n}=1)\end{array}$ & $\begin{array}{c}\text { Self-limited } \\
\text { colitis }(\mathrm{n}=6)\end{array}$ & $\begin{array}{c}\text { GVHD/IBD-like } \\
(\mathrm{n}=1)\end{array}$ \\
\hline \multicolumn{7}{|l|}{ Endoscopic appearance } \\
\hline Unknown $(n=7)$ & 2 & 4 & 0 & 0 & 1 & 0 \\
\hline Normal $(n=17)$ & 5 & 5 & 1 & 0 & 5 & 1 \\
\hline Ulcers/edema/erythema $(n=8)$ & 2 & 0 & 5 & 1 & 0 & 0 \\
\hline \multicolumn{7}{|l|}{ Bowel preparation } \\
\hline No preparation/NPO $(n=2)$ & 1 & 0 & 1 & 0 & 0 & 0 \\
\hline GoLytely $(n=16)$ & 2 & 6 & 3 & 1 & 3 & 1 \\
\hline Phospho soda $(n=2)$ & 1 & 0 & 1 & 0 & 0 & 0 \\
\hline Unknown $(n=7)$ & 3 & 2 & 0 & 0 & 2 & 0 \\
\hline Fleets enema $(n=4)$ & 1 & 1 & 1 & 0 & 1 & 0 \\
\hline Tap water enema $(n=1)$ & 1 & 0 & 0 & 0 & 0 & 0 \\
\hline
\end{tabular}

NPO: nil per os (withhold oral food and fluids); IBD: inflammatory bowel disease; GVHD: graft-vs-host disease.

Table 2 Spectrum and extent of histologic changes in cases and controls

\begin{tabular}{|c|c|c|c|c|c|c|c|c|}
\hline & \multicolumn{4}{|c|}{ Patients on $M M F$ (number of patients $=32$ ) } & \multicolumn{4}{|c|}{ Control group (number of patients $=8$ ) } \\
\hline & 0 & + & ++ & +++ & 0 & + & ++ & +++ \\
\hline Inflammation & 20 & 5 & 6 & 1 & 7 & 1 & 0 & 0 \\
\hline Crypt distortion & 11 & 12 & 7 & 2 & 8 & 0 & 0 & 0 \\
\hline Cryptitis & 23 & 5 & 4 & 0 & 8 & 0 & 0 & 0 \\
\hline Apoptosis & 19 & 6 & 6 & 1 & 7 & 0 & 1 & 0 \\
\hline Mucin depletion & 10 & 15 & 5 & 2 & 6 & 1 & 1 & 0 \\
\hline Paneth cell metaplasia & 22 & 5 & 4 & 1 & 8 & 0 & 0 & 0 \\
\hline Crypt loss & 30 & 1 & 1 & 0 & 8 & 0 & 0 & 0 \\
\hline
\end{tabular}

Biopsies were obtained from the right and left colon in 14 patients, from the left colon only (descending, sigmoid and/or rectum) in 7 patients and were taken randomly (site unspecified) in 11 patients. The average number of biopsy pieces per endoscopy was 8 (range 2-31). In patients in whom mucosal abnormalities were seen at endoscopy, histologic abnormalities were confined to those sites, whereas in the remaining cases the changes were diffuse and there was no correlation of the biopsy site with either the severity of changes or the histologic patterns. There was no specific correlation between mycophenolate mofetil dose and type of organ transplant and type of bowel preparation with the extent, pattern or severity of histologic changes. Owing to the small number of cases in each category, no statistical analysis was performed.

Pathologic changes were present in $23(23 / 40$, $58 \%$ ) patients, whereas biopsies in the remaining 17 patients appeared normal (Table 2). Apoptosis and architectural distortion were seen more frequently in patients receiving mycophenolate mofetil as compared to the control group. Six $(6 / 32,19 \%)$ patients showed increased apoptosis at the crypt bases without significant inflammation, very closely resembling colonic graft-vs-host disease (Figure 1). Nine $(9 / 32,28 \%)$ patients showed an inflammatory

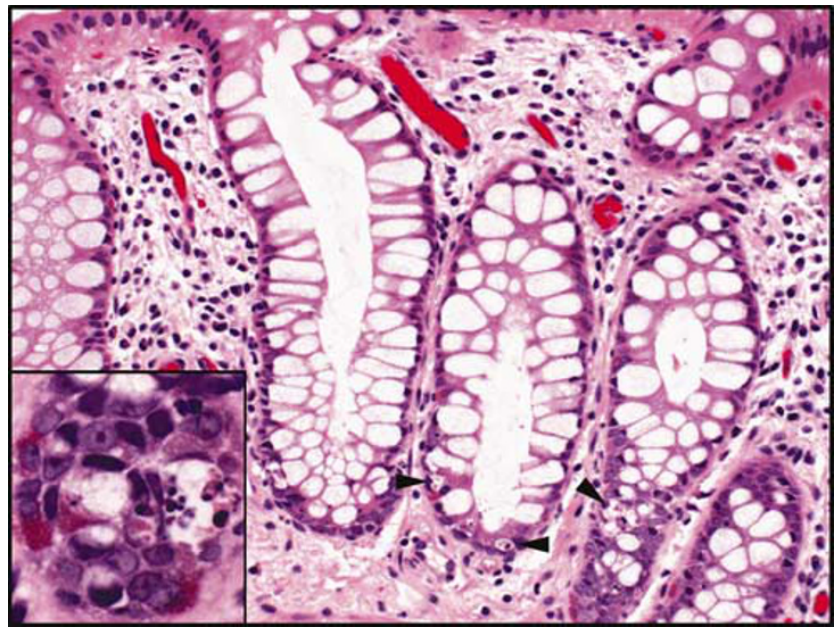

Figure 1 Graft-vs-host disease-like pattern. The crypts show increased apoptosis at the bases (arrows). The overall crypt architecture is preserved and there is no increase in lamina propria inflammatory infiltrate. Inset shows close-up of enterocyte apoptosis.

bowel disease-like pattern with architectural distortion $(9 / 9,100 \%)$, crypt abscesses $(4 / 9,44 \%)$, Paneth cell metaplasia $(7 / 9,78 \%)$ and crypt loss $(2 / 9,22 \%)$. Shortening of crypts with formation of subcryptal 

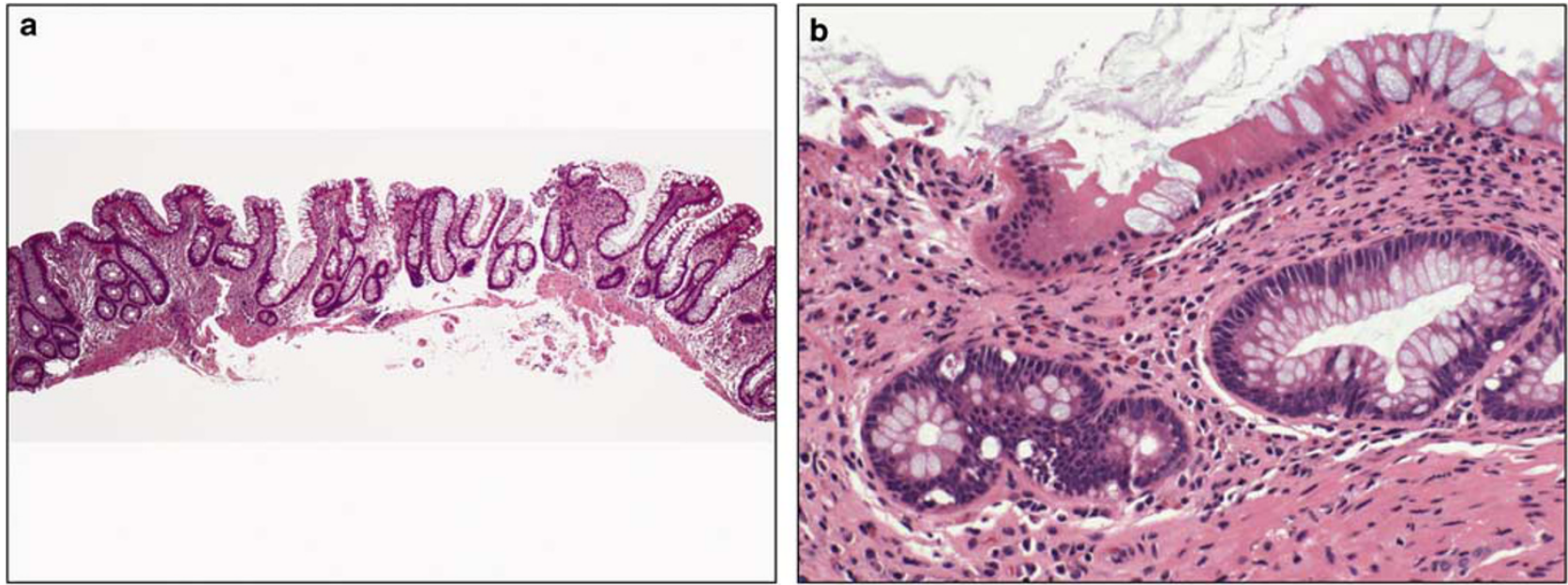

C

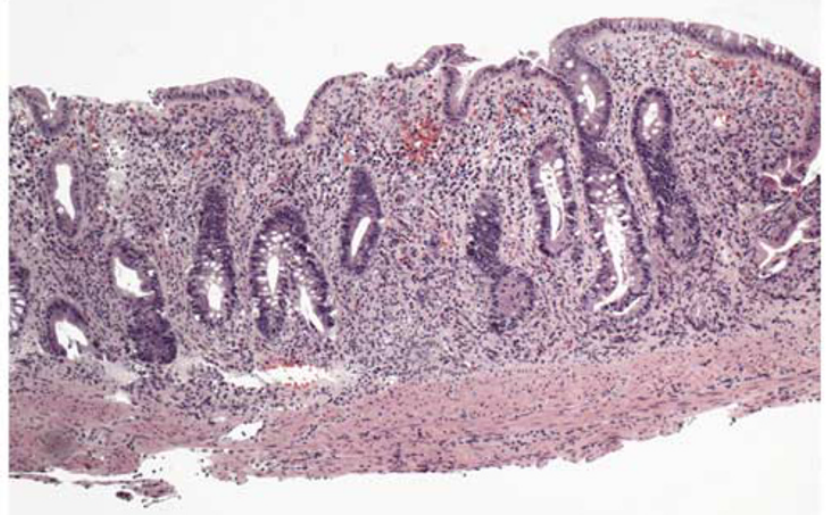

Figure 2 Inflammatory bowel disease-like pattern. (a) Architectural distortion of glands with moderate mucin depletion is seen. A mild inflammatory infiltrate is present in the lamina propria. (b) Another case showing markedly distorted crypts, crypt loss and mild increase in crypt enterocyte apoptosis mimicking both graft-vs-host disease and inflammatory bowel disease. Note that the inflammatory infiltrate is generally milder in most cases compared to typical cases of inflammatory bowel disease; however, in some cases (c) the inflammatory infiltrate can be more dense.

spaces was noted in all cases; however, lamina propria inflammatory infiltrates were milder (mostly 1 to $2+)(8 / 9,89 \%)$ when compared to typical inflammatory bowel disease cases (Figure 2a and b). One patient showed increased apoptosis in parts of the biopsy mimicking graft-vs-host disease, in addition to inflammatory bowel disease-like changes. In addition, $5(5 / 32,16 \%)$ patients showed a self-limited colitis-like pattern with mild lamina propria inflammatory changes with cryptitis and no architectural distortion (Figure 3). One patient $(3 \%)$ showed changes typical of mucosal ischemia (Figure 4). The remaining biopsies were either normal or had only nonspecific changes that included mucin depletion, rare branching crypts or mucosal edema and were considered near normal.

In comparison, 8 of the 40 patients were not taking mycophenolate mofetil and were on a combination of prednisone, tacrolimus, sirolimus and/or cyclophosphamide. Only 1 of these 8 cases (13\%) showed a mild increase in apoptosis of the crypt epithelium and the pattern was considered graft-vs-host disease-like. The remaining cases were histologically unremarkable (Table 3).

In 25 of the 32 patients on mycophenolate mofetil $(78 \%)$, the colonoscopy findings were available (Table 2). Of these, 17 (68\%) patients appeared to have normal mucosa at the time of the procedure. The remaining 8 (32\%) patients showed edema, aphthous ulcers and/or friable mucosa. The majority of biopsies that showed graft-vs-host disease-like changes were associated with endoscopic mucosal abnormalities on colonoscopy; however, such changes were also seen in the absence of any endoscopic abnormalities (Table 2). None of the patients had other systemic manifestation of graft-vs-host disease, eg, skin lesions or liver involvement.

In $15(47 \%)$ of the patients on mycophenolate mofetil therapy, mycophenolate mofetil was either withdrawn or used in reduced dosages that resulted in improvement of the symptoms. In 8 cases, the 

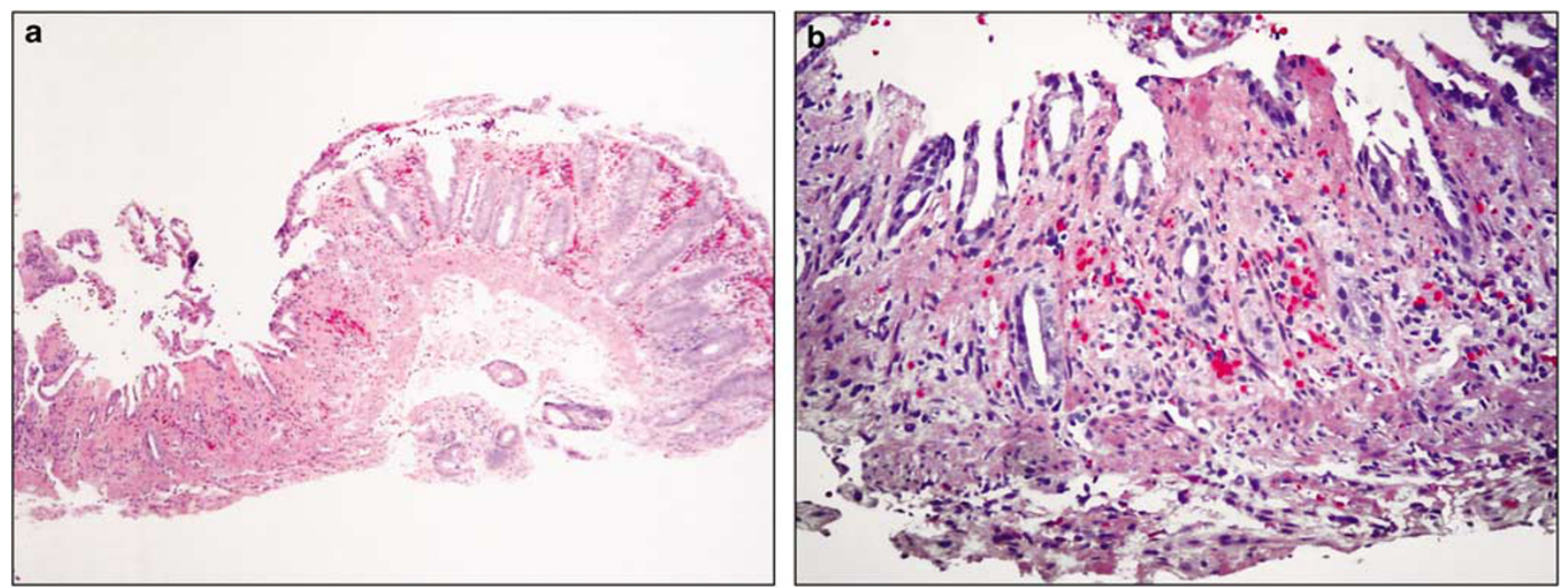

Figure 3 Ischemia-like pattern. Crypt loss and atrophy along with mucin depletion are prominent. The lamina propria is devoid of significant inflammation. The remaining areas of this biopsy showed preserved crypts with no inflammation or architectural distortion. (a) Low power and (b) high power.
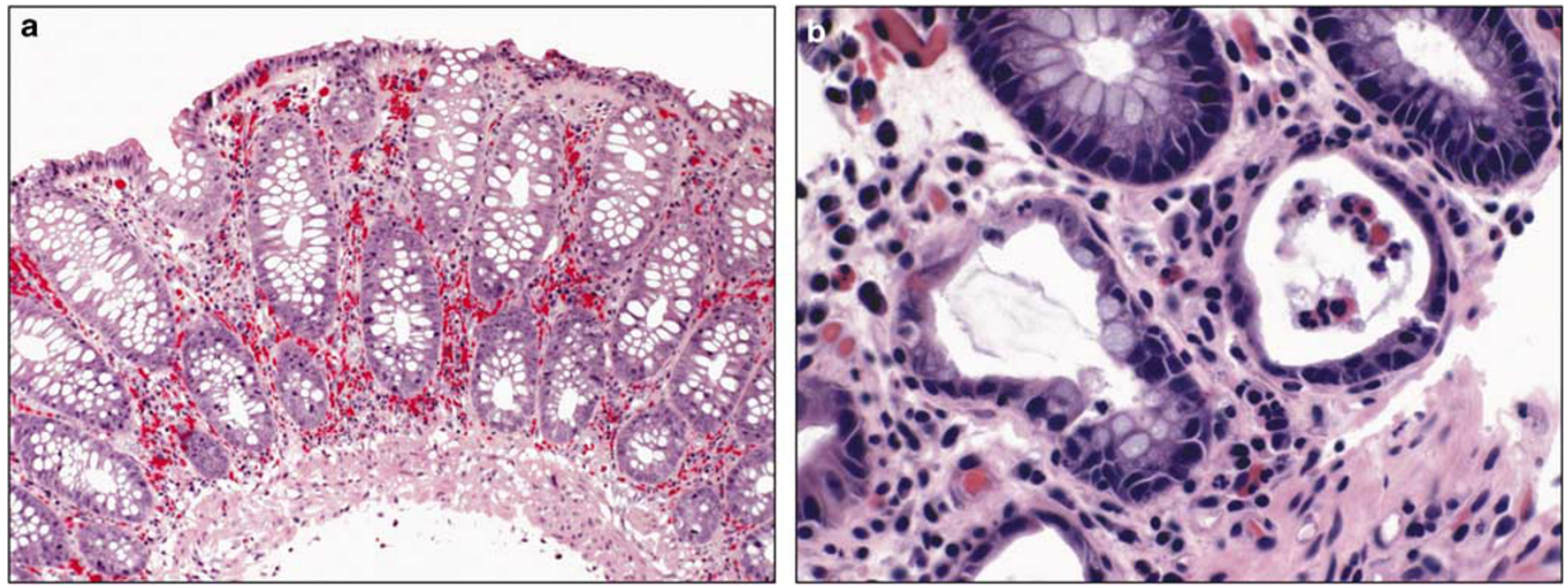

Figure 4 Self-limited pattern. (a) The lamina propria shows an increased number of inflammatory cells containing many neutrophils. The crypt architecture is preserved. (b) Neutrophilic infiltration of the crypts is seen.

Table 3 Various histologic patterns in cases and controls

\begin{tabular}{lcccccr}
\hline & Normal/near normal & IBD & GVHD & Ischemia & Self-limited colitis & GVHD/IBD-like \\
\hline MMF & 10 & 9 & 6 & 1 & 5 & 1 \\
No MMF & 7 & 0 & 1 & 0 & 0 & 32 \\
Total & 17 & 9 & 7 & 1 & 5 & 8 \\
\hline
\end{tabular}

diarrhea completely resolved following reduced dosage/withdrawal of mycophenolate mofetil. In 13 patients, the drug was continued with continuation of the diarrhea. Follow-up on the remaining patients is not available at present. Biopsies following improvement in symptoms were performed on two patients. In one patient with near normal histology on the initial biopsy, the follow-up biopsy performed 9 months later remained similar. The second patient with graft-vs-host disease-like changes on follow-up biopsy 1 month later showed near normal histology. 


\section{Discussion}

This study represents the largest report to date of the spectrum of histologic changes associated with mycophenolate mofetil therapy. Previous case reports and a small series have shown that mycophenolate mofetil can produce histologic changes in the bowel that can mimic inflammatory bowel disease, graft-vs-host disease, self-limited colitis and ischemia. ${ }^{6-8}$ The case report by Kim and Park ${ }^{8}$ described a 49-year-old woman with diarrhea following renal transplantation who was found to have mucosal ulcers, hyperemia and edema during colonoscopy. Findings on biopsy were consistent with ischemic colitis. In the only other series looking at the histologic features induced by mycophenolate mofetil, a graft-vs-host disease-like pattern in patients taking mycophenolate mofetil was described. ${ }^{6}$ In this study, 20 renal transplant patients on mycophenolate mofetil were examined. ${ }^{6}$ The parameters looked at in this study were crypt enterocyte apoptosis, architectural disarray, epithelial cell atypia, lamina propria inflammation, intraepithelial lymphocytes, neuroendocrine cells, mononuclear cell apoptosis and mucosal vascular injury. Patients receiving mycophenolate mofetil were compared to bone marrow/peripheral blood stem cell transplant patients with documented graft-vs-host disease, other renal transplant patients without mycophenolate mofetil in their regimen, patients with inflammatory bowel disease or infectious colitis and normal colon from patients with no known disease. Our study showed similar findings in renal, pancreatic, heart and liver transplant patients. In our study, we also found histologic changes that simulated inflammatory bowel disease and self-limited colitis. These changes appear to occur more in patients on mycophenolate mofetil than those in the control group.

The distinction between mycophenolate mofetilassociated graft-vs-host disease-like changes and true graft-vs-host disease is of great clinical significance. Although graft-vs-host disease in solid organ transplant patients is uncommon (the exact incidence is unknown), it can occur in patients 1-11 weeks following transplantation. ${ }^{9}$ Solid organs contain immunologically competent cells from the donor that are transplanted into the recipient, which can then initiate an immune response against the recipient. In solid organ transplantation, the incidence of graft-vs-host disease is highest following small bowel transplant (about $5 \%$ ) possibly due to a large number of donor lymphocytes in the transplanted organ. In practice, however, most cases of solid organ-associated graft-vs-host disease are seen following liver transplantation ${ }^{10}$ because small bowel transplantation is limited to a few centers across the country.

The treatment for mycophenolate mofetil toxicity is to decrease the dosage of mycophenolate mofetil, and subsequently evaluate whether the patient's symptoms resolve. In contrast, the treatment for graft-vs-host disease is to increase immunosuppression with corticosteroids and, depending on the severity of symptoms, add other immunosuppressive agents. ${ }^{11}$ Other less commonly used treatments for graft-vs-host disease include infusion of activated host lymphocytes ${ }^{12}$ and photopheresis. ${ }^{13}$ Mycophenolate mofetil is frequently used in allogeneic bone marrow/peripheral blood stem cell transplant patients and graft-vs-host disease is estimated to occur in 33-64\% of cases depending on the HLA compatibility of the donor and recipient. ${ }^{14}$ In one study of 740 allogeneic bone marrow transplant patients with acute graft-vs-host disease, it was found that $81 \%$ of patients had skin involvement, $54 \%$ had gastrointestinal involvement and $50 \%$ had liver involvement. ${ }^{15}$ Skin involvement is usually seen initially. Thus, correlation with other manifestation of graft-vs-host disease (skin or liver involvement) may be helpful in some cases; however, one may have to rely entirely on the gastrointestinal biopsies to resolve the issue. Another factor that can potentially complicate this situation is that apoptosis can be seen in colonic biopsies as a result of drugs such as nonsteroidal anti-inflammatory agents ${ }^{16}$ and bowel preparation, especially phospho soda. ${ }^{17}$ In our study, we could not find any correlation with the type of bowel preparation used and histologic changes, although in clinical practice this needs to be kept in the differential diagnosis.

One case report described mucosal ischemia-like changes in a 49-year-old renal transplant patient, which resolved after withdrawal of mycophenolate mofetil. ${ }^{8}$ The patient in our study is similar and had known underlying cardiovascular disease that could have led to mucosal ischemia. The follow-up on this patient revealed an acute lower gastrointestinal bleed that also supports the findings of mucosal ischemia. The patient's mycophenolate mofetil dose was not adjusted and there was no recurrence of mucosal ischemia following supportive treatment for the gastrointestinal bleed. On the basis of our observation and the other reported case, we believe this might represent true mucosal ischemia, and not necessarily represent mycophenolate mofetil-induced mucosal injury. We suggest that more studies are needed before ascribing ischemic changes to mycophenolate mofetil toxicity.

The differentiation of mycophenolate mofetilinduced changes from infectious colitis (self-limited colitis pattern) could also be difficult. Examination of the stool was not performed in the six cases of acute/self-limited colitis pattern in our study. In two cases with a self-limited pattern of colitis, diarrhea resolved after mycophenolate mofetil was removed from the treatment regimen. In another patient with diarrhea, mycophenolate mofetil was withheld and gradually restarted after the symptoms resolved. There was no change in mycophenolate mofetil dose in two of the patients with a self-limited colitis 
pattern and no follow-up was available on the last patient. The differentiation of mycophenolate mofetil-induced changes from inflammatory bowel disease may pose fewer difficulties in most cases, as the inflammation in the lamina propria is generally milder compared to typical cases of inflammatory bowel disease. Endoscopic appearance and prior history of inflammatory bowel disease, if available, would also be helpful in some cases. One patient with graft-vs-host disease-like changes showed resolution of the histologic changes after a month; however, since completion of the study, we have seen a few cases with persistent inflammatory bowel disease-like changes for months (up to 12 months) despite some clinical improvement. Whether these findings represent new onset inflammatory bowel disease, where the histology has been modified by immunosuppressive therapy or merely a response to chronic mycophenolate mofetil-induced injury remains speculation at present.

The major clinical limitations of mycophenolate mofetil are related to hematopoietic suppression and gastrointestinal intolerance. Although the exact mechanism by which mycophenolate mofetil induces changes in gastrointestinal mucosa is unknown, it has been hypothesized to be caused by MPA. Gastrointestinal epithelial cells, as well as other cells in the body, are partially dependent on the de novo pathway for growth and replication. ${ }^{18}$ The biopsy changes described in our patients were seen with mycophenolate mofetil, an immediate release formulation of MPA. Whether these changes would also be seen with enteric-coated mycophenolate sodium, which delays MPA release until the small intestine, has yet to be investigated. Some studies have shown an improvement in gastrointestinal adverse events with enteric-coated mycophenolate sodium. ${ }^{19}$

In summary, in our experience, currently there is no definite way to distinguish between mycophenolate mofetil toxicity and graft-vs-host disease, and future studies are needed to more clearly address these issues. Bowel preparation artifacts and selflimited colitis may also be difficult to differentiate from mycophenolate mofetil-induced colitis in some cases. The recognition of the association between mycophenolate mofetil and histologic changes mimicking graft-vs-host disease is important, given that graft-vs-host disease is not only a significant differential diagnostic consideration in allogeneic bone marrow transplant patients, but can also lead to major life-threatening complications. Similarly, in some patients, mycophenolate mofetil-induced changes could lead to an erroneous diagnosis of inflammatory bowel disease or self-limited colitis. Increased awareness of the histologic spectrum of mycophenolate mofetil-induced changes is necessary for the pathologist to avoid diagnostic errors. Further studies that evaluate the mycophenolate mofetilinduced changes in cases of bone marrow transplant patients are needed to resolve these issues.

\section{References}

1 Ziriakus M. Hoffman-La Roche receives FDA approval to market CellCept for preventing liver transplant rejection. Transplant News; advance online publication August 28, 2000 (ISSN: 1090-4964).

2 European Mycophenolate Mofetil Cooperative Study Group. Placebo-controlled study of mycophenolate mofetil combined with cyclosporin and corticosteroids for prevention of acute rejection. Lancet 1995;345:1321-1325.

3 Lee WA, Gu L, Miksztal AR, et al. Bioavailability improvement of mycophenolic acid through amino ester derivatization. Pharm Res 1990;7:161-166.

4 Behrend M. Adverse gastrointestinal effects of mycophenolate mofetil: aetiology, incidence and management. Drug Saf 2001;24:645-663.

5 Pietruck F, Abbud-Filho M, Vathsala A, et al. Conversion from mycophenolate mofetil to enteric-coated mycophenolate sodium in stable maintenance renal transplant patients: pooled results from three international, multicenter studies. Transplant Proc 2007; 39:103-108.

6 Papadimitriou JC, Cangro CB, Lustberg A, et al. Histologic features of mycophenolate mofetil-related colitis: a graft versus-host disease-like pattern. Int J Surg Pathol 2003;11:295-302.

7 Dalle IJ, Maes BD, Geboes KP, et al. Crohn's-like changes in the colon due to mycophenolate? Colorectal Dis 2005; 7:27-34.

8 Kim HC, Park SB. Mycophenolate mofetil-induced ischemic colitis. Transplant Proc 2000;32:1896-1897.

9 Jamieson NV, Joysey V, Friend PJ, et al. Graft-versushost-disease in solid organ transplantation. Transpl Int 1991;4:67-71.

10 Gulbahce HE, Brown CA, Wick M, et al. Graft-vs-host disease after solid organ transplant. Am J Clin Pathol 2003;119:568-573.

11 Higman MA, Vogelsang GB. Chronic graft versus host disease. Br J Haematol 2004;125:435-454.

12 Kuball J, Theobald M, Ferreira EA, et al. Control of organ transplant-associated graft-versus-host-disease by activated host lymphocyte infusions. Transplantation 2004;78:1774-1779.

13 Zic JA, Miller JL, Stricklin GP, et al. The North American experience with photopheresis. Ther Apher 1999;3:50-62.

14 Sullivan KM, Agura A, Anasetti F, et al. Chronic graftversus-host disease and other late complications of bone marrow transplantation. Semin Hematol 1991; 28:250-259.

15 Martin PJ, Schoch G, Fisher L, et al. A retrospective analysis of therapy for acute graft-versus-host disease: initial treatment. Blood 1990;76:1464-1472.

16 Lee FD. Importance of apoptosis in the histopathology of drug related lesions in the large intestine. J Clin Pathol 1993;46:118-122.

17 Parfitt J, Driman D. Pathological effects of drugs on the gastrointestinal tract: a review. Human Pathol 2007;38:527-536.

18 Allison AC, Eugui EM. The design and development of an immunosuppressive drug, mycophenolate mofetil. Springer Semin Immunopathol 1993;14:353-380.

19 Calvo N, Sanchez-Fructuoso AI, Conesa J, et al. Renal transplant patients with gastrointestinal intolerability to mycophenolate mofetil: conversion to entericcoated mycophenolate sodium. Transplant Proc 2006; 38:2396-2397. 